\title{
COVID-19 and HIV testing: different viruses but similar prejudices and psychosocial impacts
}

\author{
Janet Michel $^{1} \mathbb{0}$, Raphael Stuber ${ }^{1}$, Martin Müller ${ }^{1}$, Annette Mettler ${ }^{1}$, Hansjakob Furrer $^{2}$, Rashida A Ferrand ${ }^{3}$, \\ Aristomenis K Exadaktylos', Wolf E Hautz', Thomas C Sauter ${ }^{1}$
}

1 Department of Emergency Medicine, Inselspital, Bern University Hospital, 2 Department of Infectious Diseases, Inselspital, University Hospital, 3 Department of Clinical Research, Faculty of Infectious and Tropical Diseases, London School of Hygiene and Tropical Medicine; Biomedical Research and Training Institute

Keywords: triage, covid-19, hiv, psychosocial, stigma

https://doi.org/10.29392/001c.21403

\section{Journal of Global Health Reports}

Vol. 5, 2021

\begin{abstract}
Background
Severe acute respiratory syndrome coronavirus 2 (SARS-CoV-2) high infectivity and perceived substantial fatality rates are causing negative psychosocial effects, including the increased psychiatric and economic burden. Research has demonstrated that a severe diagnosis triggers various responses in a person, including depression, sense of hopelessness, shame, and self-destructive behaviours. This manuscript explores the reasons why people did not follow the recommendations to be tested for SARS-CoV-2.
\end{abstract}

\section{Methods}

A mixed study design, sequential explanatory study was carried out from March-Dec 2020, based on an online COVID-19 symptom checker. Quantitative and qualitative data were collected. Video interviews were held with Key Informants $(n=19)$, who were selected purposefully from the online tool users' group that consented to the study.

\section{Results}

Among 176 users of the online triage tool, 150 (85\%) followed the recommendations and 26 (15\%) did not. The reasons people did not test for SARS-CoV-2 emerged as fivefold: i) improved symptoms, ii) the cost of test, iii) fear of a painful test procedure, iv) test kit shortages, and $v$ ) fear of a positive SARS-CoV-2 test result.

\section{Conclusions}

Of the reasons why people did not test, fear of a positive SARS-CoV-2 result remains unaddressed in our view. Integrating pre- and post-test counselling into SARS-CoV-2 testing strategies, similarly as done for HIV-testing, seems warranted to address this problem.

Dealing with a life-threatening illness exhibits different reactions in people. This is of special interest in the situation where the whole world is faced with an unprecedented challenge of severe acute respiratory syndrome coronavirus 2 (SARS-CoV-2). Its high infectivity and perceived substantial fatality rates may cause psychosocial effects including psychiatric and economic burdens. ${ }^{1-3}$ Research has demonstrated that a severe and potentially fatal diagnosis triggers various responses in a person, including depression, denial, hopelessness, shame, self-destructive behaviours, and insomnia, ${ }^{4}$ most of which have also been associated with COVID-19. This is further exemplified by the daily reports on the number of infected, those at the intensive care units and deceased. ${ }^{5-7}$ Some studies have revealed that the cumulative approach to tracking COVID-19 increases fear and anxiety among the public. ${ }^{5}$ The fear COVID-19 engenders in people ought to be acknowledged for the battle to be won. ${ }^{8}$

Corona phobia, meaning mass fear of the virus, has been associated with psycho-social illnesses after the infection. ${ }^{1}$ Three common strategies to combat the spread of SARS$\mathrm{CoV}-2$ are lockdowns, quarantine and isolation. The diagnosis of COVID-19 accompanied with the required isolation, has been revealed to cause acute panic, anxiety, depression, stigmatization, and even elements of racism in some instances. ${ }^{1}$ Front line health care workers have not been spared either, with some suffering from burn out, fear of transmitting infection to family members, fear of contracting infection and even developing a post-traumatic stress disorder. ${ }^{1,2}$ Finally, the breadth of media coverage, the "infodemic", further contributed to this situation and aggravated psychosomatic conditions like depression and anxiety, amongst all socio-economic strata, possibly to a greater extent than the virus itself, ${ }^{1}$ making this epidemic a real public health issue.

Social stigma, blame and loneliness associated with COVID-19 have also been reported. Social stigma has been 
associated with avoidance of testing. ${ }^{9}$ It has been defined as an attribute which is deeply discrediting, reducing a person in the widest sense of that word. ${ }^{9,10}$ It is most closely felt in the index cases, and its detrimental effects are visible not only in fear, but also in blame. ${ }^{10}$ One of the most devastating effects of stigmatization on the societal level is the avoidance of testing, rising from the shame and even selfrejection, a special form of internalized stigma. ${ }^{10}$

The aim of this study was to explore and understand if OFTT users followed the recommendations given and the reasons they followed or disregarded these. In addition, we explored the psychosocial consequences of a COVID-19 diagnosis that might be preventing people from testing, and impacting their mental health, too.

\section{METHODS}

\section{CONTEXT AND INTERVENTION}

The Department of emergency medicine at Inselspital, Bern University Hospital, developed an online forward triage tool (OFTT).This OFTT was, to the best of our knowledge, one of the first of its kind in Switzerland. ${ }^{11}$ Recommendations to test or not to test were made in accordance with frequently changing guidelines about testing published by the federal office of public health (FOPH). The aim of the OFTT was to provide the users with recommendations about testing in Switzerland during the first COVID-19 wave.

We carried out the mixed study design to explore the effects and utility of the OFTT. The rationale for collecting both quantitative and qualitative data in one study is that neither method is sufficient by itself; only a combination of both approaches can yield a holistic picture of a phenomenon. Quantitative and qualitative methods complement each other and may yield advantages that are not accessible in a single-approach study. This manuscript explores why people did not follow the recommendations to test for COVID-19, while the utility and effects of an online COVID-19 symptom checker were published separately. ${ }^{12}$

\section{DATA COLLECTION AND ANALYSIS}

The survey instrument was pilot tested, by inviting the emergency room physicians to fill it out. After achieving satisfactory results of the piloting, we offered the survey to the target sample. The data collection began on March 2, 2020 and ended on Dec 10, 2020. Quantitative data was collected from all OFTT users between March 2 and May 12, 2020. The database complied with the Swiss laws of collecting personal health related data. Participants that consented to a follow-up study were invited to take part in an online survey to ascertain if they indeed followed the online tool recommendations and why they had done or not done so.

\section{QUANTITATIVE DATA AND SAMPLING}

Quantitative data was analysed in Stata ${ }^{\circledR} 16.1$ (StataCorp, The College Station, Texas, USA). Descriptive statistics for all variables were computed as mean, standard deviation, and range or frequency as determined by the type of the data.

\section{QUALITATIVE DATA}

Qualitative data was collected between 17 August 2020 and 10 Dec 2020. Qualitative data was collected in order to supplement the quantitative findings, as well as understand the reasons why participants followed or disregarded recommendations. The sample was derived from OFTT users who had taken part in the online survey, and had additionally consented to follow-up qualitative interviews, in a multistage sampling scheme. In order to ensure that participants from all age groups were included, purposeful and quota sampling were performed. Video interviews were held with Key Informants $(n=19)$. The interviews were audio recorded and transcribed verbatim.

\section{CENTRAL QUESTION}

\section{"Why did people that got a recommendation to test for COVID-19 not do so?”}

\section{QUALITATIVE DATA ANALYSIS}

An iterative, deductive and inductive approach was utilized to analyse data. Transcribed data were coded thematically with the aid of MAXQDA2018 (VERBI software, Berlin). Measures to ensure trustworthiness of data were taken. For dependability, the data collection process is well described and two experienced qualitative researchers kept reflexive journals through-out data collection and analysis phases. Debriefings after end of interviews were conducted daily and two researchers experienced in qualitative methods coded the data independently for themes before reaching consensus. To ensure confirmability, we discussed findings with experts in the field of HIV, infectious diseases and telemedicine, and incorporated their responses. Participants, context and process of analysis have been described in detail to enhance transferability. Interviews were only stopped after reaching data saturation. After the $9^{\text {th }}$ key informant interview, themes started to be repetitive with no new themes emerging.

\section{KEY INFORMANTS AND SAMPLE SIZE}

The sample was derived from OFTT users that had taken part in the online survey and had further consented to follow-up qualitative interviews-multistage sampling. In order to ensure that participants from all age groups were included, purposeful and quota sampling were done (Table 1).

\section{ETHICS APPROVAL}

The local ethics committee of Canton Bern, Switzerland, deemed this project a quality evaluation study, and waived the need for full ethical review (Req-2020-00289) on March 23, 2020.

\section{RESULTS \\ OUANTITATIVE RESULTS}

There were 6,272 users of the OFTT between March 2 and 
Table 1. Key informant demographics

\begin{tabular}{|l|l|l|l|l|l|}
\hline Age groups & $18-29$ & $30-45$ & $46-64$ & $65+$ & Total \\
\hline Males & 1 & 2 & 3 & 4 & 10 \\
\hline Females & 2 & 2 & 4 & 1 & 9 \\
\hline Total & 3 & 4 & 7 & 5 & 19 \\
\hline
\end{tabular}

Table 2. Summary of themes in qualitative analysis

\begin{tabular}{|c|c|c|}
\hline Theme & Category & Unit meaning \\
\hline 1. People did not test when the symptoms improved & Natural recovery process & - improved symptoms \\
\hline 2. People did not test due to the cost of test & Economic factor & $\begin{array}{l}\text { - cost of COVID-19 test deterred } \\
\text { some }\end{array}$ \\
\hline $\begin{array}{l}\text { 3. People did not test due to fear of a painful test } \\
\text { procedure (rumours) }\end{array}$ & $\begin{array}{l}\text { Information, education, and } \\
\text { communication (IEC) }\end{array}$ & $\begin{array}{l}\text { - misinformation-rumours that } \\
\text { test is very painful }\end{array}$ \\
\hline 4. People did not test because of test kit shortages & $\begin{array}{l}\text { Supply chain challenges } \\
\text { Pandemic preparedness }\end{array}$ & $\begin{array}{l}\text { - test kit shortages-testing ca- } \\
\text { pacity }\end{array}$ \\
\hline $\begin{array}{l}\text { 5. People did not test due to fear of a positive test } \\
\text { result }\end{array}$ & $\begin{array}{l}\text { Psychological readiness to accept } \\
\text { diagnosis }\end{array}$ & $\begin{array}{l}\text { - fear of a positive test and its } \\
\text { consequences } \\
\text { - prefer not to know }\end{array}$ \\
\hline
\end{tabular}

May 12, 2020; 176 of them consented to participate in a follow-up study. The participants' mean age was 50.1 years (SD 15.4, range 18-82). There were 101 (57.4\%) women among them, with 31 (17.6\%) of the elderly, aged 65 and above. The vast majority followed the given recommendations given, 150 subjects (85\%) and 26 subjects (15\%) did not.

\section{QUALITATIVE FINDINGS}

Most of the participants revealed that test kit shortages experienced during the early phases of the pandemic, the cost of the test at the time, improved symptoms, and fear of a painful test (disinformation) were some of the reasons people did not test (Table 2).

Most of the participants also expressed fear and when probed revealed the following:

With all the consequences, one thinks it's better not to test, I would rather not know and continue working - Key informant 8

Fear of a positive result is the reason. Many people are afraid of a positive COVID 19 test. That is what I heard from my friends and colleagues. Many said they would rather stay at home, isolate and that's it, rather than deal with a positive test - Key informant 18

\section{DISCUSSION}

This study identified a framework of five reasons of failure to follow recommendations to be tested for COVID-19. These reasons included: i) the lack of testing when the symptoms improved, ii) problems due to the cost of testing iii) fear of a painful testing procedure, iv) kit shortages, and v) fear of a positive test result. The most salient result of this study is the emergence of fear and psychological readiness to receive such a diagnosis.

The majority of the reasons revealed are addressed easily and have been solved in most countries. Lack of testing in the face of improved symptoms is a natural disease course pattern, and therefore presents an understandable situation where no further diagnostic procedure may seem warranted. Shortage of test kits and fear of a painful testing are all barriers that can be addressed through a functional supply chain, information, education and open communication. Financial challenges associated with the cost of testing have already been addressed by making the SARS-CoV-2 test free in many countries, including Switzerland.

Contrary to this, the fear of a positive SARS-CoV-2 result remains largely unaddressed. Several previous studies have reported prevalent psychiatric conditions following COVID-19.8,13,14 Any new and potentially life-threatening disease in a population seems to be associated with psychosocial and behavioural issues that need to be taken into account. ${ }^{4}$ The fear of a positive test result and resultant consequences have also been previously reported. 13,14 The psychological readiness of patients, to deal with a diagnosis that is new and considered severe seems to fuelling this. We feel this is important particularly as rapid tests are being rolled out. 13

\section{ISOLATION AND SOCIAL DISTANCING}

Strategies currently in use to curb the spread of the virus are isolation, immediately post diagnosis and quarantine after exposure, and these seem to exacerbate fear in some, leading to mental health challenges in the long run. Our research revealed that dealing with fear and the psychologi- 


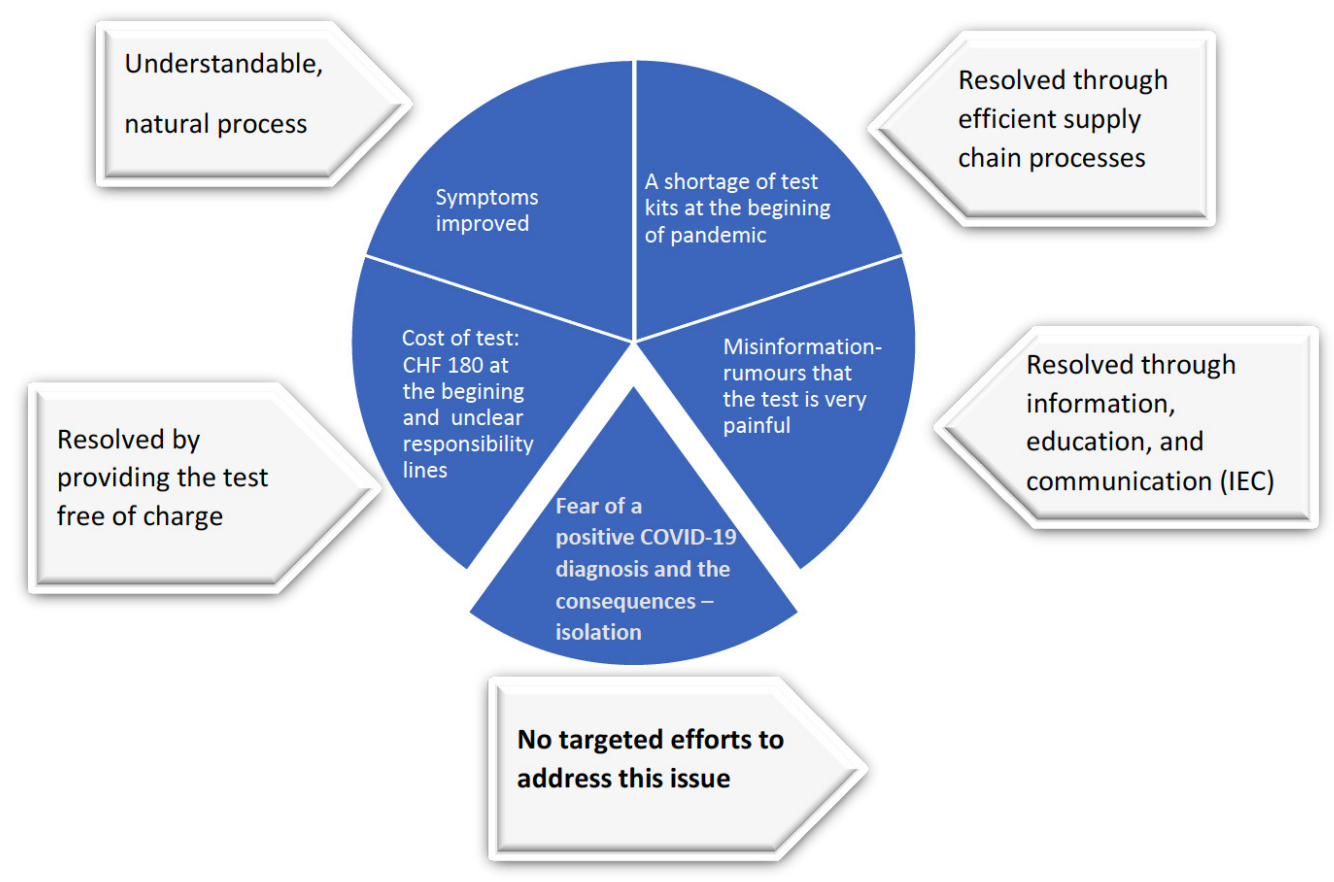

Figure 1. The framework of the reasons for non-testing and possible measures to tackle them

cal readiness of patients to receive a COVID-19 diagnosis, are important but seemingly neglected issues. ${ }^{14}$ If almost 1 in 5 people diagnosed with COVID-19 are diagnosed with a mental illness within 3 months of their positive test, ${ }^{15}$ with prevalent neuro-psychiatric sequelae, ${ }^{16}$ then our perception and the importance of this infection must be reconsidered. This is further aggravated by the negative consequences of loneliness and isolation, in addition to the disease itself, ${ }^{17,18}$ suggesting that this is a real public health priority at a population level. Psychosocial crisis prevention and interventions should therefore be urgently developed and expanded as a public health measure. ${ }^{1}$ Telemedicine interventions could be employed to address both the pandemic and infodemic needs. ${ }^{1,14}$ The need for psychosocial preparedness in future pandemics cannot be over emphasized. $^{1}$

\section{ARE WE SEEING THIS FOR THE FIRST TIME?}

No; a diagnosis with a life-threatening illness is known to be associated with psychosocial behaviours. ${ }^{4}$ This was especially true for cancer; preparing the patients for a possible cancer diagnosis, providing information, life expectancy, effect of cancer on other life aspects and involvement of close relatives, were associated with lower incidents of depression and positive patient outcomes. ${ }^{19}$

\section{LEARNING FROM HIV TESTING}

In the early 80s, HIV was a fatal disease that was associated with substantial stigma and blame, providing the grounds for a direct comparison with COVID-19. How did we cope back then? Pre and post-test counselling were introduced after realizing that many patients did not manage to cope well with HIV diagnosis. ${ }^{20}$ The existing evidence shows that subjects who experienced pre- and post-counselling were able to cope with their illness better, and were more likely to look after their health and protect others from infection. ${ }^{14,20-22}$ In support of our findings, parallels between HIV and COVID-19 have also been mentioned elsewhere. ${ }^{23}$ It could easily be that this kind of COVID-19 diagnosis impact is currently underestimated.

\section{PRE- AND POST-TEST COUNSELLING AS A PUBLIC HEALTH TOOL TO FIGHT COVID-19 RELATED FEAR}

Pre and post counselling could be done by a nurse or GP with the help of digital tools. Telemedicine, in the form of phone or video consultations, have already proven effective during this pandemic. ${ }^{24}$ We therefore propose to offer pre and post-test counselling - a cheap, affordable, accessible, and sustainable public health tool, as an additional strategy to address COVID-19 related fear to the patients. Additional lessons could also be drawn from HIV second generation surveillance, through strategic concentration of information and resources, that help build up an informative picture of changes in the COVID-19 pandemic overtime. ${ }^{25}$

\section{STRENGTHS AND LIMITATIONS}

Our OFTT was one of the first COVID-19 OFTT to be set up in Switzerland. The insights gained here could be used to inform and improve future COVID-19 and other pandemic tools. While many OFTTs are collecting quantitative data only, we employed a mixed study design, yielding a holistic picture, going beyond reporting the proportion of OFTT users that does not follow recommendations to test. This information is invaluable in improving the utility of COVID-19 and other OFTTs as well as patient outcomes.

Online tool users do not necessarily have to be focused 
on those with a disease. Worried people usually search for symptoms online to ascertain whether or not to seek medical care. At the same time, others might have used the tool for test runs, simply to acquaint themselves with the tool itself or COVID-19 expected signs and symptoms, as this information was unknown and scarce at the beginning of the pandemic. One limit of our study could be the low participation rate in the follow-up study. That could mean that the population that consented to study might not be representative of all online OFTT users. In order to make our OFTT available to as many people as possible, to minimize barriers to use and to comply with data protection regulations, we have nevertheless refrained from a mandatory follow-up survey.

The analogy of COVID and HIV has some inherent limitations. For example, unlike COVID, the stigma associated with HIV is not only rooted in the direct and indirect effects of the disease itself, but also in the primarily sexual mode of transmission. Secondly, HIV diagnosis implies living with the virus lifelong and taking lifelong treatment with effects on quality of life. While data on long-COVID is still emerging, the majority of the COVID-19 cases on the other hand, are usually milder, with the virus expected to clear within three months at most. Nevertheless, we believe that this analogy can help highlight similar psychosocial impacts between HIV and COVID-19 which might lead us to applicable solutions and learned lessons from the past.

\section{CONCLUSIONS}

A novel, perceived life-threatening disease in a population is associated with psycho-social and behavioural issues that need to be taken into account. Fear of a positive SARSCoV-2 testing result remains largely unaddressed. This is clearly seen in the previous comment: "Respect the fear individuals have about COVID-19. Acknowledge it. Listen to what they have to say. Support them. Be patient and compassionate. And we can overcome the fear and defeat COVID-19 together." (December 2020). ${ }^{8}$ Integrating pre- and post-test counselling into SARS-CoV-2 testing strategies, as was done for HIV-testing, may be a useful tool to address COVID-19 related fear.

\section{ACKNOWLEDGEMENTS}

Emergency telemedicine at University of Bern, Switzerland is supported by an endowed professorship by Touring Club Switzerland.

\section{PAPER CONTEXT}

A new and potentially or perceived life-threatening disease in a population seems to be associated with psycho-social and behavioural issues that need to be taken into account. Our study revealed that fear of a positive COVID-19 test result and resultant consequences, can prevent people from testing. This raises the question of the psychological readiness of patients, to deal with a diagnosis that is new and perceived as fatal. This paper highlights psychosocial consequences of a COVID-19 diagnosis that might be preventing people from testing and affecting their mental health too.

\section{DATA AVAILABILITY STATEMENT}

Raw data were generated at Insel University Hospital. Derived data supporting the findings are available from corresponding author (JM) and co-author (MM).

\section{FUNDING}

Swiss National Science Foundation (Project ID: 196615).

\section{AUTHORSHIP CONTRIBUTIONS}

JM, RS, MM, AM, FH, WEH, TCS designed the research study. JM, RS, MM, AM, FH, RAF, AKE, WEH, TCS wrote the paper and contributed to subsequent drafts. All authors commented and approved the final manuscript.

\section{COMPETING INTERESTS}

The manuscript is partially funded by the Swiss National Science Foundation (Project ID:196615). The funder has no influence on the content of the manuscript or the decision to publish it. TCS holds the endowed professorship for emergency telemedicine at the University of Bern, Switzerland. The funder has no influence on the research performed, the content of the manuscript or any decision to publish. All other author have completed the Unified Competing Interest form at www.icmje.org/coi_disclosure.pdf (available upon request from the corresponding author), and declare no conflicts of interest.

\section{CORRESPONDENCE TO:}

Janet Michel

Department of Emergency Medicine

Inselspital, University Hospital, University of Bern, 3010, Switzerland

Submitted: February 08, 2021 GMT, Accepted: March 03, 2021

GMT 


\section{REFERENCES}

1. Dubey S, Biswas P, Ghosh R, et al. Psychosocial impact of COVID-19. Diabetes Metab Syndr. 2020;14(5):779-788. doi:10.1016/j.dsx.2020.05.035

2. Wu P, Fang Y, Guan Z, et al. The Psychological Impact of the SARS Epidemic on Hospital Employees in China: Exposure, Risk Perception, and Altruistic Acceptance of Risk. Can J Psychiatry. 2009;54(5):302-311.

3. Varatharaj A, Thomas N, Ellul MA, et al. Neurological and neuropsychiatric complications of COVID-19 in 153 patients: a UK-wide surveillance study. Lancet Psychiatry. 2020;7(10):875-882. doi:10.1 016/S2215-0366(20)30287-X

4. Psychological Trauma. Fam Psychol Assoc. Published December 21, 2017. Accessed November 28, 2020. https://fampsy.org/psychological-trauma/

5. Alasousi LF, Alhammouri S, Alabdulhadi S. Anxiety and media exposure during COVID-19 outbreak in Kuwait. medRxiv. Published online September 6, 2020. doi:10.1101/2020.08.24.20180745

6. Coronavirus Update (Live): 83,889 Cases and 2,867 Deaths from COVID-19 Wuhan China Virus Outbreak - Worldometer. Accessed February 28, 2020. https://w ww.worldometers.info/coronavirus/

7. BAG. BAG-Situation-schweiz-und-international. Accessed March 18, 2020. https://www.bag.admin.ch/ bag/de/home/krankheiten/ausbrueche-epidemien-pa ndemien/aktuelle-ausbrueche-epidemien/novel-cov/s ituation-schweiz-und-international.html\#73793215

8. Shira D. To defeat Covid-19, we must acknowledge the fear it engenders. STAT. Published December 9, 2020. Accessed December 10, 2020. https://www.statn ews.com/2020/12/09/to-defeat-covid-19-we-must-ack nowledge-the-fear-it-engenders/

9. Dowdy DW, Zwerling AA, Stennett A, et al. Measuring Stigma to Assess the Social Justice Implications of Health-Related Policy Decisions: Application to Novel Treatment Regimens for Multidrug-Resistant Tuberculosis. MDM Policy Pract. 2020;5(1). doi:10.1177/2381468320915239

10. Abdelhafiz AS, Alorabi M. Social Stigma: The Hidden Threat of COVID-19. Front Public Health. 2020;8. doi:10.3389/fpubh.2020.00429

11. Hautz WE, Exadaktylos A, Sauter TC. Online forward triage during the COVID-19 outbreak. Emerg Med J. Published online December 2020. doi:10.1136/ emermed-2020-209792
12. Effects and utility of an online forward triage tool during the SARS-CoV-2 pandemic: patient perspectives. JMIR Prepr. Accessed January 4, 2021. ht tps://preprints.jmir.org/preprint/26553

13. Person B, Sy F, Holton K, et al. Fear and Stigma: The Epidemic within the SARS Outbreak. Emerg Infect Dis. 2004;10(2):358-363. doi:10.3201/eid1002.030750

14. Strathdee SA, Martin NK, Pitpitan EV, Stockman JK, Smith DM. What the HIV Pandemic Experience Can Teach the United States About the COVID-19 Response. J Acquir Immune Defic Syndr. 2021;86(1):1-10. doi:10.1097/OAI.0000000000002520

15. Bidirectional associations between COVID-19 and psychiatric disorder: retrospective cohort studies of 62354 COVID-19 cases in the USA. The Lancet Psychiatry. Published online 2020. doi:10.1016/S221 $\underline{\text { 5-0366(20)30462-4 }}$

16. Erausquin GA de, Snyder H, Carrillo M, Hosseini AA, Brugha TS, Seshadri S. The chronic neuropsychiatric sequelae of COVID-19: The need for a prospective study of viral impact on brain functioning. Alzheimer's Dement. Published online 2021:1-9. doi:10.1002/alz.12255

17. Strang P, Bergström J, Martinsson L, Lundström S. Dying From COVID-19: Loneliness, End-of-Life Discussions, and Support for Patients and Their Families in Nursing Homes and Hospitals. A National Register Study. J Pain Symptom Manage. 2020;60(4):e2-e13. doi:10.1016/j.jpainsymman.2020.0 $\underline{7.020}$

18. Wu B. Social isolation and loneliness among older adults in the context of COVID-19: a global challenge. Glob Health Res Policy. 2020;5(1):27. doi:10.1186/s412 56-020-00154-3

19. Psychological responses of patients receiving a diagnosis of cancer. Annals of Oncology. Accessed November 28, 2020. https://www.annalsofoncology.or g/article/S0923-7534(19)63901-3/fulltext

20. Information NC for B, Pike USNL of M $8600 \mathrm{R}$, MD B, Usa 20894. PRE-TEST AND POST-TEST SERVICES. World Health Organization; 2015. Accessed December 15, 2020. https://www.ncbi.nlm.nih.gov/books/NBK31 $\underline{6035 /}$

21. Kanekar AS. HIV/AIDS Counseling Skills and Strategies: Can Testing and Counseling Curb the Epidemic? Int J Prev Med. 2011;2(1):10-14. 
22. Chippindale S, French L. HIV counselling and the psychosocial management of patients with HIV or AIDS. BMJ. 2001;322(7301):1533-1535.

23. Millett GA. New pathogen, same disparities: why COVID-19 and HIV remain prevalent in U.S.

communities of colour and implications for ending the HIV epidemic. J Int AIDS Soc. 2020;23(11):e25639. doi:10.1002/jia2.25639
24. Gray L, Wong-Wylie G, Rempel G, Cook K. Expanding Qualitative Research Interviewing Strategies: Zoom Video Communications. Qual Rep. 2020;25(5):1292-1301.

25. WHO. Guidelines for second generation HIV surveillance. WHO. Accessed March 1, 2021. https://w ww.who.int/hiv/pub/surveillance/2013package/modul e1/en/ 